\title{
Twin-induced hardening in extruded Mg alloy AM30
}

\author{
Hua Qiao $^{1}$, X.Q. Guo ${ }^{1,2}$, A.L. Oppedal ${ }^{3}$, H. El Kadiri ${ }^{4}$, P.D. Wu ${ }^{1}$, and S.R. Agnew ${ }^{5, *}$ \\ ${ }^{1}$ Department of Mechanical Engineering \\ McMaster University, Hamilton, Ontario, Canada \\ ${ }^{2}$ State Key Laboratory for Geomechanics and Deep Underground Engineering \\ China University of Mining and Technology, Xuzhou, Jiangsu 221008, China \\ ${ }^{3}$ Center for Advanced Vehicular Systems, \\ Mississippi State University, Starkville, Mississippi, U.S.A. \\ ${ }^{4}$ Department of Mechanical Engineering, \\ Mississippi State University, Starkville, Mississippi, U.S.A. \\ ${ }^{5}$ Department of Materials Science and Engineering, \\ University of Virginia, Charlottesville, Virginia, U.S.A.
}

\begin{abstract}
The mechanical anisotropy of extruded Mg alloy AM30 was recently investigated, both experimentally and numerically (Oppedal et al. [1]). The authors highlighted the need to include cases of intermediate levels of twinning as a critical validation, in addition to the limiting cases where the contribution of twinning is dominant or negligible. However, further scrutiny of the results revealed that the experimental data employed in that study were still inadequate to constrain all necessary parameters in the crystal plasticity models. In particular, the parameters describing the prismatic slip mechanism were under-constrained. In the present study, additional experimental data were obtained to provide the necessary constraint. Based upon these experimental results, clearer conclusions can be drawn about the requirements of a crystal plasticity model, which must accurately account for strong tension-compression asymmetry as well as anisotropy in both strength and strain hardening behavior. In addition to the previously employed Viscoplastic Self-Consistent (VPSC) model, with the Predominant Twin Reorientation (PTR) scheme, the Elastic Visco-Plastic Self-Consistent (EVPSC) model, with the recently developed Twinning and De-Twinning (TDT) description, is applied to simulate the uniaxial response along arbitrary directions. It is demonstrated that accounting for the initial texture and calibrating the EVPSC-TDT model using uniaxial tension and compression along the extrusion direction permits prediction of the strength anisotropy and strain hardening behavior along arbitrary straining directions.
\end{abstract}

Keywords: twinning; crystal plasticity; anisotropy; strain hardening; hexagonal close packed *Corresponding author. Tel.: +1 (434) 924-0605; Fax: +1 (434) 982-5660;

E-mail: agnew@virginia.edu 


\section{Introduction}

Most of the present experimental data were recently employed (Oppedal et al. [1]) to constrain and validate a study of crystal plasticity modeling of a textured $\mathrm{Mg}$ alloy, which emphasized two main points.

1) A physically-based model should not only describe limiting cases, where the contribution of twinning is either maximized or minimized. Rather, it should also be able to predict cases with intermediate levels of twinning.

2) In order to accurately accomplish the above goal, the interactions between slip- and twinning-based deformation must be accurately taken into account.

The authors go on to suggest that a dislocation density-based approach, which incorporates the effects of transmutation (defined below), more accurately accounts for the observed behavior than previously employed models (Oppedal et al. [1]). This experimental data has been revisited, additional experiments have been performed on the same material, and greater clarity regarding the requirements of a crystal plasticity model has yielded additional conclusions. The paper is divided into two sections.

In Section 1, it is demonstrated that it is possible to obtain equally good descriptions of the plastic anisotropy of a textured $\mathrm{Mg}$ alloy extrusion using a number of different empirical descriptions of dislocation- and twinning-induced strain hardening. The basic foundation of each of the models is a visco-plastic self-consistent (VPSC) polycrystal plasticity model, which has been described in numerous prior papers (Molinari et al. [2]; Lebensohn and Tomé [3]; Proust et al. $[4,5])$. This portion of the paper illustrates the caution with which we should draw conclusions regarding the benefits of one modeling strategy over another. In section 2, one of the conclusions of the original work is further emphasized. In short, it is stressed that adequate experimental data 
must be incorporated in order to constrain all of the parameters in the model.

\section{Background}

"Transmutation" is a term employed to describe what happens when a twin boundary advances into a previously dislocated matrix or when dislocations from the matrix propagate through the twin. It should be obvious that the dislocations cannot maintain their same Burgers vectors, etc., a fact which Basinski et al. [6] highlighted many years ago in the context of face centered cubic (FCC) alloys and which Niewczas [7] recently adapted to hexagonal close packed (HCP) metals by employing a geometric construct termed the correspondence matrix originally introduced by Bevis and Crocker [8]. Salem et al. [9] provided experimental support for the concepts of Basinski et al. [6], especially the fact that the twins would be harder than the surrounding matrix. El Kadiri and Oppedal [10] proposed a crystal plasticity theory for latent hardening based upon these transmutation reactions. Oppedal et al. [1,11] went on to demonstrate the efficacy of the transmutation concept through crystal plasticity modeling. In fact, Wang and Agnew [12] have confirmed one of the proposed transmutation reactions by performing TEM analysis of dislocation interactions with the $\{10.2\}$ type twin boundary in $\mathrm{Mg}$ alloy, AZ31. Indeed, Yoo [13] long ago suggested that twin boundaries in HCP metals would be more or less effective for obstructing dislocation activity, depending upon the relative orientations of the specific slip system and twin variant.

The above arguments are largely geometric, and admittedly fail to capture key interfacial details which have come to light through the atomistic simulation work of Serra and Bacon [14], Wang et al. [15], and El Kadiri et al. [16]. Figure 1 presents a schematic illustration of the interactions between dislocations and twin boundaries. Basal glide dislocations within the matrix are colored red, while basal dislocations within the twin are colored yellow. Note that the basal 
planes within the matrix and twin are nearly perpendicular to one another. The boundary reactions yield a number of products, including "transmuted" dislocations which cross the twin boundary (colored blue) as well as residual dislocations within the interface (shown in green, which incidentally serve as twinning disconnections whose glide will result in twin growth or shrinkage), and residual disclinations (which are not shown here in the interest of simplicity, but are detailed in reference [16]). It is not the purpose of the present work to advance the theory of dislocation-twin interactions. Rather, it is to highlight the need for more detailed considerations, so that future modeling efforts rely less on empirical data. However, the present data will reinforce the notion that the effect of twinning on subsequent slip can be potent, and recent studies ( $\mathrm{Li}$ and $\mathrm{Ma}$ [17]; Tu et al. [18]; Wang and Agnew [12]) of dislocations in around the twins continue to elucidate this issue.

\section{Experimental methods}

The material of this study is the same AM30 magnesium alloy (chemical composition, wt.\%: 2.54 Al, 0.4 Mn, 0.018 Zn, 0.03 Fe, $0.008 \mathrm{Si}, 0.011 \mathrm{Cu}, 0.025 \mathrm{Ce}, 0.005 \mathrm{Ni}$, balance Mg) employed previously (Oppedal et al. [1]). It was received from Timminco, Ltd. in the form of a $200 \mathrm{~mm}$ diameter billet extruded from a $450 \mathrm{~mm}$ diameter as-cast ingot. The extrusion operated at $570^{\circ} \mathrm{C}$ and at a $0.3 \mathrm{~ms}^{-1}$ ram speed. Reconstructed pole figures of the starting material, obtained by neutron diffraction, are presented in Figure 1 of the paper by Oppedal et al. [1]. The typical extruded 'rod texture' of HCP metals is observed, with $<10.0$. $>$ fiber parallel to the extrusion axis, which places the (00.1) basal poles perpendicular to the extrusion axis. Samples for uniaxial mechanical testing were prepared using electrical discharge machining (EDM). The compression samples were 10 -mm diameter right cylinders, whereas the tension samples had a gage section with $6 \mathrm{~mm}$ diameter and $24 \mathrm{~mm}$ gage length. 
Typically, studies like this have involved samples deformed along axes of symmetry. The early study of Kleiner and Uggowitzer [19] and the original study of AM30 by Oppedal et al. [1] are exceptions to this rule, since the compression samples were cut parallel to a variety of different directions. The four directions indicated in Figure 2 were selected to provide conditions where varying levels of twin activity could be observed. More recently, some of the authors have employed a similar approach in studying thick Mg alloy, AZ31, plate (Guo et al. [20,21]).

During the present reconsideration, the modeling revealed that substantial twinning is observed for all four of these directions, and that the flow stress is not controlled by prismatic slip in any of them. Thus, after publication of the original manuscript (Oppedal et al. [1]), a series of additional tensile tests were performed along the extrusion direction (ED) and orthogonal it. The results of the latter showed large uncertainty, presumably because they were not carefully excised along radial (ERD) directions. Thus, only the ED tensile test data is considered here. The flow stress during ED tension will be revealed to have been controlled by prismatic slip, and the activity of twinning is minimal (it is a limiting case).

All the tension and compression tests were performed using an Instron 5869 at room temperature and at a strain rate of $0.001 \mathrm{~s}^{-1}$. Molykote ${ }^{\circledR}$ lubricant was used on the compression test platens, and no barreling was observed in the tested specimens. Generally, the compression samples failed by fracture/shear localization, at plastic strain of about -0.15 . The reported test data are from samples that were interrupted prior to failure.

\section{Modeling approach}

Section 1: In this section, we employ the previously published data and illustrate that a range of modeling strategies can satisfactorily describe the observed behavior. The basic foundation of 
each approach is the visco-plastic self-consistent (VPSC) polycrystal plasticity code, which has been described in numerous prior papers (Molinari et al. [2]; Lebensohn and Tomé [3]; Proust et al. $[4,5])$. Specifically, two different intermediate linearization schemes (affine and $N_{\text {eff }}=10$ ), which have been demonstrated to be most effective in prior studies (Wang et al. [22]; Qiao et al. [23]), are compared. These two are denoted as "intermediate" because they fall in between the extreme cases of equilibrium first (Sachs) and compatibility first (Taylor). Further, they are intermediate to the self-consistent schemes based upon tangent or secant linearization (Lebensohn and Tomé [3]; Hutchinson [24]). It will be shown that either of these linearization strategies will work. Because it has been described numerous times in the literature, we will not reiterate the basics of crystal plasticity, such as the use of the Schmid law to the activation of slip and twinning (e.g., Van Houtte [25]) or the use of a power law relationship between shear strain rate and the resolved shear stress (e.g., Asaro and Needleman [26]). Rather, we will focus on the specific elements which pertain to twinning and which were evaluated in the present study.

With respect to accounting for the crystallographic reorientation due to twinning, two different strategies are employed. The first is the predominant twin reorientation $(P T R)$ scheme introduced by Tomé et al. [27]. In this approach, the amount of shear strain, $\gamma_{i}$, accommodated by the $i^{\text {th }}$ twin variant within a grain is monitored and the corresponding twin volume fraction, $f_{i}$, is computed in the normal way (e.g., Van Houtte [25]),

$$
f_{i}={ }_{i}{ }^{t w}
$$

where $\gamma^{t w}$ denotes the characteristic twinning shear of the mode in question. When the accumulated twin volume fraction within a grain, $f^{a c c}=\sum_{i}^{n} f_{i}$, exceeds a threshold value,

$$
V^{t h}=\min \left(1.0, A_{1}+A_{2} \frac{V^{e f f}}{V^{a c c}}\right) \text {, }
$$


the entire grain is reoriented to the orientation of the most active twin variant. Thus, the model introduces two statistical variables, which are volume weighted averages over all the grains, $g$, in the entire polycrystal: the accumulated twin fraction, $V^{a c c}=\sum_{g} w_{g} f_{g}^{a c c}$, and effective twinned fraction $V^{e f f}=\sum_{g} w_{g}^{t w}$ (i.e., that which has been reoriented). Additionally, two material constants $A_{1}$ and $A_{2}$ are introduced which define the volume fraction at which the first grain is reoriented and the rate at which $V^{e f f}$ will "catch up" to the actual value, $V^{a c c}$, once reorientation has commenced. These parameters can be employed to tune the length of the "twinning plateau" predicted by the model as well as the sharpness with which the plateau ends.

An advantage of the PTR approach is that it is computationally efficient. There is no increase in the number of grains that must be tracked by the code. However, there are two disadvantages that may become significant if strain path changes are of interest, especially at small or intermediate strain levels (c.f. Proust et al. [4]). The first is the fact that the volume fraction of reoriented material $V^{e f f}$ does not at all points properly correlate with the amount of twinning which has taken place $V^{a c c}$ (though it does match at higher strain levels). The second aspect is that not every activated twin variant is present in the final simulated "microstructure," since only the predominant twin variant is respected by the model. This may be fine for cases in which a single twin variant dominates. However, there may be cases when many variants are observed to occur.

The second approach has been described at length in recent publications, Wang et al. [28, 29] and Qiao et al. [30], and is denoted the twinning-detwinning (TDT) model. In summary, the TDT model assumes that a grain has four potential operations associated with twinning and detwinning. Operation A is twin nucleation and initiates a twin band or 'child'. Operation B is a propagation of the child into the parent grain. Operations A and B increase the twin volume 
fraction and thus correspond to twinning. Operation $\mathrm{C}$ is a propagation of the parent into the child. Operation D splits the twin band and decreases the twin volume fraction through retwinning. Operations $\mathrm{C}$ and $\mathrm{D}$ decrease the twin volume fraction and thus correspond to detwinning.

Unlike the PTR model, where the original grain orientation is transformed to the twin orientation, the TDT model generates a new "grain" every time a new twin variant is activated. This approach suffers a computational penalty for this grain proliferation. In principle, the number of grains could be 7 times the original number (for the HCP case and considering only primary twinning of the most common mode, $\{10.2\}<10.1>$, with one new grain for each of the 6 variants). The evolution of twin volume fraction associated with $i^{\text {th }}$ twin variant is governed by:

$$
\dot{f}_{i}=f_{0}\left(\dot{f}_{i}^{A}+\dot{f}_{i}^{C}\right)+f^{\beta}\left(\dot{f}_{i}^{B}+\dot{f}_{i}^{D}\right)
$$

where $f_{0}$ is the volume fraction of the parent, i.e. $f_{0}=1 f^{a c c}, f^{\beta}$ is the volume fraction of a given twin variant $\beta$, and the superscripts $\mathrm{A}, \mathrm{B}, \mathrm{C}$ and $\mathrm{D}$ represent for Operations $\mathrm{A}, \mathrm{B}, \mathrm{C}$ and $\mathrm{D}$, respectively. It is important to note that twin nucleation by Operation A has to be driven by the stress state of the parent, since there is not yet a twin (child). In this case, twinning is activated by a driving force, i.e. the resolved shear stress on the twinning plane and in the twinning direction, $\tau_{i}^{g}=\mathbf{P}_{i}: \boldsymbol{\sigma}^{g}$, is calculated using the true stress tensor $\boldsymbol{\sigma}^{g}$ acting on the parent grain, $g$, and the Schmid tensor $\mathbf{P}_{i}$ associated with the $i^{\text {th }}$ twin variant. Similarly, once a twin is formed, it is obvious that Operation D, taking place inside a twinned domain, has to be driven by the stress state of the twinned region or the child, $\boldsymbol{\sigma}^{t w}$.

In reality, the growth or shrinkage of an existing twin is in fact driven by shear stress on the twin boundary (TB) itself. The approach adopted is an approximation of this reality. For 
example, since Operation B is a propagation of the child into the parent, it seems appropriate to activate it by the resolved shear stress $\tau_{i}^{t w}=-\mathbf{P}_{i}: \boldsymbol{\sigma}^{t w}$ calculated from the child stress state. The negative sign respects the fact that variant $i$ generates opposite shear in the twin to that which it creates within the parent. Similarly, Operation $\mathrm{C}$ is driven by $\tau_{i}^{g}=-\mathbf{P}_{i}: \boldsymbol{\sigma}^{g}$. These relationships do not violate the fact that twinning is a polar mechanism. Rather they refine our understanding by illustrating that twinning is only polar during initiation (a twin cannot be generated by stress in the anti-twinning direction). Once the twin is formed, it can accommodate shear in either twinning (Operations A and B) or the anti-twinning sense (Operations C and D).

The TDT model considers a twin as a new grain in the sense that a new ellipsoid is added to the 1-site self-consistent formulation. The orientation of the new grain is initially related to that of the parent through the crystallographic twin relation (for the $\{10.2\}$ twinning mode, the c-axis is rotated by $86.3^{\circ}$ about the $\langle 11.0\rangle$ directions), and the volume fraction associated with the new grain is given by relations (1) at the end of the first straining step in which the twin variant is activated. From this point onward, the twin only "interacts" with its parent through the exchange of volume fraction (Eq. 3) and the fact that the model enforces the same critical shear stresses for twinning on both the twin and its parent. In short, in this approximation, there is no special enforcement equilibrium or compatibility between the twin and parent. Rather, the twin and parent are forced to satisfy equilibrium and compatibility with the self-consistent homogeneous effective medium (HEM), which surrounds each of them. Thus, the model is designed to enforce a sort of pseudo-twin boundary (TB) between the twin and its parent grain.

A final aspect relates to the fact that it is rare to find a grain that is fully twinned. Thus, the TDT model invokes equation (2) to terminate twinning in a given grain, once the accumulated volume fraction reaches a threshold value. This aspect of the model may be viewed as 
accounting for sharp increase in the resistance to continued twin growth by the surrounding medium, once the threshold value is reached. As was the case for the PTR model, the threshold parameters $\left(A_{1}\right.$ and $\left.A_{2}\right)$ control the length of the yield plateau and the sharpness with which it ends. It is imagined that these parameters will have a strong grain size dependence to account for observed changes in the flow curve shape for cases of twinning dominated flow in samples of different grain sizes (c.f. Barnett et al. [31]).

Section 2: In this section, new experimental data (ED tension) is employed to constrain the parameters, which govern the prismatic slip mode. This additional constraint creates challenges for the aforementioned VPSC modeling strategies. Recently, some of the present authors illustrated that the Elastic Visco-Plastic Self-Consistent (EVPSC) model of Wang et al. [32], with the TDT model can describe the yielding and strain hardening anisotropy observed experimentally for an extruded Mg alloy, ZK60, plate (Qiao et al. [30]). Notably, the model parameters were fit to the limiting cases of tension and compression along the extrusion direction, and then, the model was able to successfully predict the response along the plate transverse and normal directions. What was particularly promising was the ability to accurately predict the constitutive response under limiting cases where the straining is essentially twinning dominated and essentially devoid of twinning, as well as intermediate cases where twinning is a moderate contributor to the strain. It will not be claimed that this is the only way or the best way to model the present data. However, it will be shown to be possible to model the range of behaviors observed using the EVPSC-TDT model. There are other attractive alternatives, which might be employed, and such opportunities will be highlighted in the discussion section.

For both sections of the paper, the evolution of the slip resistance $\tau_{c r}^{\beta}$ required to activate a slip system (or twinning variant) $\beta$ is given by: 


$$
\dot{\tau}_{c r}^{\beta}=\frac{d \hat{\tau}^{\beta}}{d \Gamma} \sum_{\chi} H^{\beta \alpha}\left|\dot{\gamma}^{\alpha}\right|
$$

where $\Gamma=\sum_{\beta} \int\left|\dot{\gamma}^{\beta}\right| d t$ is the accumulated shear strain in the grain, and $H^{\beta \alpha}$ are the latent hardening coupling coefficients, which empirically account for the obstacles on system $\beta$ associated with shear rate, $\dot{\gamma}^{\alpha}$, on system $\alpha . \hat{\tau}^{\beta}$ is the threshold stress and is characterized by an extended Voce hardening model:

$$
\hat{\tau}^{\beta}=\tau_{0}^{\beta}+\left(\tau_{1}^{\beta}+h_{1}^{\beta} \Gamma\right)\left(1-\exp \left(-\frac{h_{0}^{\beta}}{\tau_{1}^{\beta}} \Gamma\right)\right)
$$

Here, $\tau_{0}, h_{0}, h_{1}$ and $\tau_{0}+\tau_{1}$ are the initial slip resistance, the initial hardening rate, the asymptotic hardening rate, and the back-extrapolated slip resistance, respectively. In some cases, the stress to initiate twinning has been assumed to be higher than that required to grow the twin once it is formed (e.g., Qiao et al. [30]). This detail was not accounted for in the present case.

The dislocation density-based hardening model advanced by Oppedal et al. [1] is not further investigated here, though it has merit, particularly for non-proportional loading scenarios. The goal here is to clarify the requirement to completely parameterize any crystal plasticity model approach and to identify the minimum requirements of the polycrystal homogenization scheme.

\section{Results and Discussion}

Section 1: Four different VPSC modeling approaches were employed to describe the compression response of extruded AM30 bar. They are the four permutations of 2 linearization strategies (Affine and $N_{\text {eff }}=10$ ) and the 2 twinning schemes (PTR and TDT). The latent hardening and Voce parameters were adjusted to obtain a best fit to the experimental data, and the results show that any of these strategies work equally well. Note that we only employ 4 
different deformation mechanisms: basal and non-basal slip of $\langle a\rangle$ dislocations, slip of $\langle\mathrm{c}+\mathrm{a}\rangle$ dislocations on second order pyramidal planes, and $\{10.2\}$ extension twinning. We do not employ $\{10.1\}$ compression or $\{10.1\}-\{10.2\}$ "double" twinning, because we do not think there is adequate experimental data to differentiate the effects of these mechanisms from the $\langle\mathrm{c}+\mathrm{a}\rangle$ slip mode (Agnew et al. [33]). This is in the spirit of making sure we have adequate experimental data to constrain all free parameters in the model. It is admitted that these (and other (ref. ReedHill and Robertson [34])) secondary twinning mechanisms do operate within $\mathrm{Mg}$ alloys, and can play a particularly important role in localization and fracture (Barnett [35]).

Table 1 presents the best-fit parameters for the hardening models (Equations 2, 4, \& 5), which were employed with the two twinning models and linearization schemes under consideration. Figures 3 and 5 present the experimental (Oppedal et al. [1]) and simulated compression curves for tests performed parallel to ED and ERD as well as two oblique directions oriented $30^{\circ}$ and $60^{\circ}$ away from the extrusion direction (see Fig. 2). Figures 4 and 6 presents the strain hardening rates as a function of the applied strain along the same directions. Regardless of the linearization scheme or the twinning model employed, the results appear equally good.

The texture evolutions, during compression parallel to ERD and at compressive strains of 0.05 (Fig. 7) and 0.11 (Fig. 8), are predicted to be very similar amongst various modeling approaches. This reinforces the notion that the predicted slip and twinning mode activities were very similar. Note that the crystals rotate in proportion with the amount of shear on each slip system and the volume fraction of crystals which are reoriented by twinning is proportional to the amount of shear accommodated by twinning (see Eq. 1). For comparison purposes, Figures 7 and 8 also present the initial texture employed in all of these calculations. It is a discrete texture comprised of $\sim 3958$ grains whose volume fractions were computed to best represent the 
experimentally measured texture in the rod (see Figure 1 of Oppedal et al. [1]). Only the texture evolution during ERD compression is presented, since the texture evolution during ED compression of a rod-textured Mg alloy is very familiar (c.f., Clausen et al. [36]). The texture evolution during compression along the oblique directions ED30 and ED60 do not provide additional insight.

Because the predicted slip and twinning mode activities are very similar between each of the VPSC modeling schemes considered (as suggested by the similar flow curve and texture evolution predictions), only the results of the VPSC-TDT, $N_{\text {eff }}=10$ model are presented in Figure 9. Not surprisingly, the results show that the slip and twinning activities are quite sensitive to the compressive loading direction. What is quite interesting and important to the present investigation is the significant activity of prismatic slip during compression along ED, right at the point where the strain hardening rate begins to sharply increase. Although the Schmid factor for prismatic slip is high in most grains of this sample loaded along ED, the extension twinning mode is similarly loaded and has a lower resolved shear resistance. Prismatic slip is being called upon right as the twinning mode is beginning to exhaust. In fact, one of the key reasons that the hardening response presented in Figures 4 and 6 is able to match so well is due to this activity of prismatic slip.

Section 2: Despite the fact that prismatic slip appears to contribute at a key point in the deformation, the VPSC results presented in Figures $3-6$ are still rather insensitive to the strength of the prismatic slip mode. It was realized that comparing with date obtained from tension along ED would provide the desired additional constraint on the selection of parameters describing prismatic slip. Figure 10 presents the results of a number of such tensile tests along with simulation results obtained using the VPSC-TDT (Fig. 10a) and EVPSC-TDT (Fig. 10b) 
models (both with the $N_{\text {eff }}=10$ linearization scheme) using parameters presented in Tables 2 and 3, respectively. Note that the strength of the prismatic slip system had to be significantly increased from the previously obtained $70 \mathrm{MPa}$ range, all the way up to $110 \mathrm{MPa}$. The <c+a> slip mode also had to be increased, to avoid activating it significantly during ED tension. Because the parameters presented in Tables 2 and 3 are considered to capture the complete range of behaviours, from minimal twinning (ED tension) to twinning dominated behaviour (ED compression presented in Figures 10 - 14), it is believed that they are more realistic than those presented in Table 1.

The high strengths of non-basal slip resulted in a challenge for the VPSC-TDT model, which is most evident in Figure 12. Note that the strain hardening rate for ED compression is predicted to be very large, once twinning is exhausted at the plastic strain of $\sim-0.06$. The predicted maximum value of strain hardening rate is over $0.5 G$ (see Fig. 12). With non-basal slip strengths high enough to model ED tension, the highly overestimated level of strain hardening of the flow curve for ED compression is predicted no matter what other model parameters were employed. The observed behaviour of strain hardening rate is associated with a composite effect where a significant volume fraction of grains are actually loading elastically, once twinning is exhausted and slower strain hardening does not recommence until the harder non-basal slip modes such as $<\mathrm{c}+\mathrm{a}>$ slip which permit c-axis deformation are activated. The VPSC model cannot account for such elastic loading, since it employs a rigid visco-plastic constitutive framework, which explains why the predicted strain hardening rate is too high. Note that elasticity adds a level of flexibility in the compatibility scheme.

In the end, the EVPSC-TDT code was employed, since it had proven successful for simulating similar behaviour previously (Qiao et al. [30]; Guo et al. [20,21]). Using the EVPSC 
code, it is expected to better predict composite behaviour in which some of the grains are essentially elastic compared with the VPSC model. In all the EVPSC-TDT simulations reported in the present paper, the room temperature elastic constants of the magnesium single crystal are taken from Simmons and Wang [37]: $C_{11}=58.0, C_{12}=25.0, C_{13}=20.8, C_{33}=61.2$ and $C_{44}=16.6$ (units of GPa). Although the EVPSC-TDT model does not perfectly describe the experimental flow curve (see Fig. 13) for ED compression and the corresponding strain hardening rate is still somewhat overestimated (see Fig. 14), it clearly provides better results than the VPSC model.

Figure 15 provides a comparison of the slip and twinning mode activities predicted by the VPSC and EVPSC approaches, when employing the model parameters presented in Tables 2 and 3, respectively. Note that both show a lower activity of the prismatic slip system during ED compression, as compared to that predicted by VPSC models employing the parameters in Table 1. Because modeling the tension data requires a high strength of the prismatic mode, its activity is suppressed, and this has the concurrent effect of enhancing the hardening rates upon the exhaustion of twinning. The reason the EVPSC model better describes the overall behavior is that elasticity provides another means of accommodating strain, when no plastic modes are available. Intensive study on the effect of elasticity on the strain hardening rate of flow curve has been carried out and the work will be reported elsewhere (Wu et al. [38]).

Figures 10b, 13, and 14 show that the EVPSC-TDT (with the $N_{\text {eff }}=10$ linearization scheme) can accurately describe the tensile and compressive yield asymmetry, anisotropy, and strain hardening behavior along arbitrary directions, including those which involve minimal (ED tension), dominant (ED compression), and intermediate (ERD, ED30, \& ED60) levels of 
twinning. The dislocation density-based model presented previously (Oppedal et al. [1]) also shows promise and future work is planned to implement this model within the EVPSC framework. Efforts will be made to incorporate insights obtained from recent TEM study of dislocation-twin boundary interactions (c.f. Wang and Agnew [12]).

Finally, it is worth to mentioning that although not shown in the present paper, our numerical results indicate that the $N_{\text {eff }}=10$ self-consistent scheme results in a better prediction than the Affine scheme, for both the VPSC-TDT and EVPSC-TDT models. This is interesting, since it was previously concluded that the VPSC-PTR model with the Affine self-consistent scheme gives the best overall performance, for a magnesium alloy AZ31B sheet (Wang et al. [22].

\section{Conclusions}

In the previous study by Oppedal et al. [1], the authors suggested that a dislocation densitybased approach with the effects of dislocation transmutation by twinning included, more accurately accounts for the observed behavior than previously employed models. These experimental data have been revisited in the current study, and it is demonstrated that it is possible to obtain equally good descriptions of the plastic anisotropy of a texture $\mathrm{Mg}$ alloy extrusion using a number of different empirical descriptions of dislocation- and twinninginduced strain hardening.

Additional experiments of tension along ED have been performed on the same material. This additional constraint creates challenges for the VPSC modeling strategies. However, it is seen that the EVPSC-TDT can generally describe the tensile and compressive yield asymmetry, anisotropy, and strain hardening behavior along arbitrary directions. In particular, the EVPSCTDT model predicts a more reasonable strain hardening rate for the case of ED compression due 
to the fact that accounting for elasticity in the model adds flexibility in the simulation compared with the VPSC model. These additional conclusions emphasize that adequate experimental data must be incorporated in order to constrain all of the parameters in polycrystal plasticity models.

\section{Acknowledgements}

H. Qiao and P.D. Wu are supported by the Natural Sciences and Engineering Research Council of Canada (NSERC). X.Q. Guo acknowledges the support of the State Key Development Program for Basic Research of China (Grant No. 2013CB227900), the Joint Funds of the National Natural Science Foundation of China (Grant No.U1261201) and China Council Scholarship (No. 201206420031). S.R. Agnew would like to thank the National Science Foundation of the U.S.A., grant number CMMI 1235259, and McMaster University for sponsoring a visiting faculty appointment, during which this work was performed.

\section{References}

[1] A.L. Oppedal, H. El Kadiri, C.N. Tomé, S.C. Vogel, M. Horstemeyer, Philos. Mag. 93 (2013) 4311-4330.

[2] A. Molinari, G.R. Canova, S. Ahzi, Acta Metall. 35 (1987) 2983-2994.

[3] R.A. Lebensohn, C.N. Tomé, Acta Metall. Mater. 41 (1993) 2611-2624.

[4] G. Proust, C.N. Tomé, G.C. Kaschner, Acta Mater. 55 (2007) 2137-2148.

[5] G. Proust, C.N. Tomé, A. Jain, S.R. Agnew, Int. J. Plasticity 25 (2009) 861-880.

[6] Z.S. Basinski, M.S. Szczerba, M. Niewczas, J.D. Embury, S.J. Basinski, Rev. Metall. 94 (1997) 1037-1043.

[7] M. Niewczas, Acta Mater. 58 (2010) 5848-5857.

[8] M. Bevis, A.G. Crocker, Proc. Roy. Soc. London A 304 (1968) 123-134.[9] A.A. Salem, S.R. Kalidindi, R.D. Doherty, S.L. Semiatin, Metall. Mater. Trans. A37 (2006) 259-268.

[10] H. El Kadiri, A.L. Oppedal, J. Mech. Phys. Solids, 58 (2010) 613-624.

[11] A.L. Oppedal, H. El Kadiri, C.N. Tomé, G.C. Kaschner, S.C. Vogel, J.C. Baird, M.F. Horstemeyer, Int. J. Plasticity, 30 (2012) 41-61.

[12] F. Wang, S.R. Agnew, Int. J. Plasticity 81 (2016) 63-86.

[13] M.H. Yoo, Trans. TMS-AIME 245 (1969) 2051-2060. 
[14] A. Serra, D.J. Bacon, Philos. Mag. 73 (1996) 333-343

[15] J. Wang, I.J. Beyerlein, C.N. Tomé, Int. J. Plasticity 56 (2014)156-172.

[16] H. El Kadiri, C.D. Barrett, J. Wang, C.N. Tomé, Acta Mater. 85 (2015) 354-361.

[17] B. Li, E. Ma, Philos. Mag. 89 (2009) 1223-1235.

[18] J. Tu, X. Zhang, J. Wang, Q. Sun, Q. Liu, C.N. Tomé, Appl. Phys. Lett. 103 (2013) 051903.

[19] S. Kleiner, P.J. Uggowitzer, Mater. Sci. Eng. A, 379 (2004) 258-263.

[20] X.Q. Guo, A. Chapuis, P.D. Wu, S.R. Agnew, Int. J. Solids Struct. 64-65 (2015) 42-50.

[21] X.Q. Guo, A. Chapuis, P.D. Wu, Q. Liu, X.B. Mao, Mater. Des. 98 (2016) 333-343.

[22] H. Wang, B. Raeisinia, P.D. Wu, S.R. Agnew, C.N. Tomé, Int. J. Solids Struct. 47 (2010) 2905-2917.

[23] H. Qiao, P.D. Wu, H. Wang, M.R. Gharghouri, M.R. Daymond, Int. J. Solids Struct.71 (2015) 308-322.

[24] J.W. Hutchinson, Proc. R. Soc. Lond. A348 (1976) 101-127.

[25] P. Van Houtte, Acta Metall. 36 (1978) 591-604.

[26] R.J. Asaro, A. Needleman, Acta Metall. 33 (1985) 923-953.

[27] C.N. Tomé, R.A. Lebensohn, U.K. Kocks, Acta Metall. Mater. 39 (1991) 2667-2680.

[28] H. Wang, P.D. Wu, C.N. Tomé, J. Wang, Mater. Sci. Eng. A 555 (2012) 93-98.

[29] H. Wang, P.D. Wu, J. Wang, C.N. Tomé, Int. J. Plasticity 49 (2013) 36-52.

[30] H. Qiao, S.R. Agnew, P.D. Wu, Int. J. Plasticity 65 (2015) 61-84.

[31] M.R. Barnett, M.D. Nave, A. Ghaderi, Acta Mater. 60 (2012) 1433-1443.

[32] H. Wang, P.D. Wu, C.N. Tomé, Y. Huang, J. Mech. Phys. Solids 58 (2010) 594-612.

[33] S.R. Agnew, D.W. Brown, C.N. Tomé, Acta Mater. 54 (2006) 4841-4852.

[34] R.E. Reed-Hill, W.D. Robertson, Acta Metall. 5 (1957) 717-727.

[35] M.R. Barnett, Mater. Sci. Eng. A464 (2007) 8-16.

[36] B. Clausen, C.N. Tomé, D.W. Brown, S.R. Agnew, Acta Mater. 56 (2008) 2456-2468. 
[37] G. Simmons, H. Wang, Single crystal elastic constants and calculated polycrystal properties, MIT Press, 1971.

[38] P.D. Wu, X.Q. Guo, H. Qiao, S.R. Agnew, D.J. Lloyd, J.D. Embury, Acta Mater., Submitted for publication, 2016. 


\section{Figure Captions:}

Figure 1. Schematic illustration of matrix dislocations (red) interacting with a twin boundary. Blue arrows indicate direction of motion of the twin boundary, which has overtaken some matrix dislocations. The possible byproducts include transmuted dislocations within the twin (blue) as well as residual interfacial defects (green). Of course there are analogous interactions that can occur with dislocations within the twins (yellow) that may seek to penetrate into the matrix. Dashed lines indicate the slip planes of dislocations of the corresponding color.

Figure 2. Schematic illustration of the four sample orientations excised from the extruded bar and tested in compression (after Oppedal et al. [1]).

Figure 3. Experimental (Oppedal et al. [1]) and VPSC-PTR simulated compression curves for tests performed parallel to the extrusion (ED) and radial (ERD) directions (left) and two oblique directions oriented $30^{\circ}$ and $60^{\circ}$ away from the extrusion direction (see Fig. 2).

Figure 4. Experimental (Oppedal et al. [1]) and VPSC-PTR simulated normalized strain hardening rates as a function of the applied strain parallel to the extrusion (ED) and radial (ERD) directions (left) and two oblique directions oriented $30^{\circ}$ and $60^{\circ}$ away from the extrusion direction (see Fig. 2).

Figure 5. Experimental (Oppedal et al. [1]) and VPSC-TDT simulated compression curves, for tests performed along the 4 directions indicated in Fig. 2.

Figure 6. Experimental (Oppedal et al. [1]) and VPSC-TDT simulated normalized strain hardening rates as a function of the applied strain, for tests performed along the 4 directions indicated in Fig. 2.

Figure 7. Crystallographic texture prior to deformation and after a compressive strain of 0.05 parallel to the radial (ERD) direction.

Figure 8. Crystallographic texture prior to deformation and after a compressive strain of 0.11 parallel to the radial (ERD) direction.

Figure 9. Deformation mechanism activity plots show how the sample orientation influences the activity of a) basal, b) prismatic, c) $\langle\mathrm{c}+\mathrm{a}>$ slip, and d) extension twinning during compression testing along the ED, ED30, ED60, and ERD, respectively. (The results of the VPSC-TDT, $N_{\text {eff }}=10$ calculation are presented as representative.)

Figure 10. Experimental as well as VPSC-TDT (a) and EVPSC-TDT (b) simulated tensile curves for tests performed parallel to the extrusion (ED).

Figure 11. Experimental (Oppedal et al. [1]) and VPSC-TDT simulated compression curves, for tests performed along the 4 directions indicated in Fig. 2. 
Figure 12. Experimental (Oppedal et al. [1]) and VPSC-TDT simulated strain hardening rates as a function of the normalized effective stress, for tests performed along the 4 directions indicated in Fig. 2.

Figure 13. Experimental (Oppedal et al. [1]) and EVPSC-TDT simulated compression curves, for tests performed along the 4 directions indicated in Fig. 2.

Figure 14. Experimental (Oppedal et al. [1]) and EVPSC-TDT simulated strain hardening rates as a function of the normalized effective stress, for tests performed along the 4 directions indicated in Fig. 2.

Figure 15. Deformation mechanism activity plots for tension (a \& c) and compression (b $\&$ d) strained along ED for the VPSC and EVPSC calculations, respectively. Each selfconsistent scheme employs the TDT twinning model and the $N_{\text {eff }}=10$ linearization scheme. 


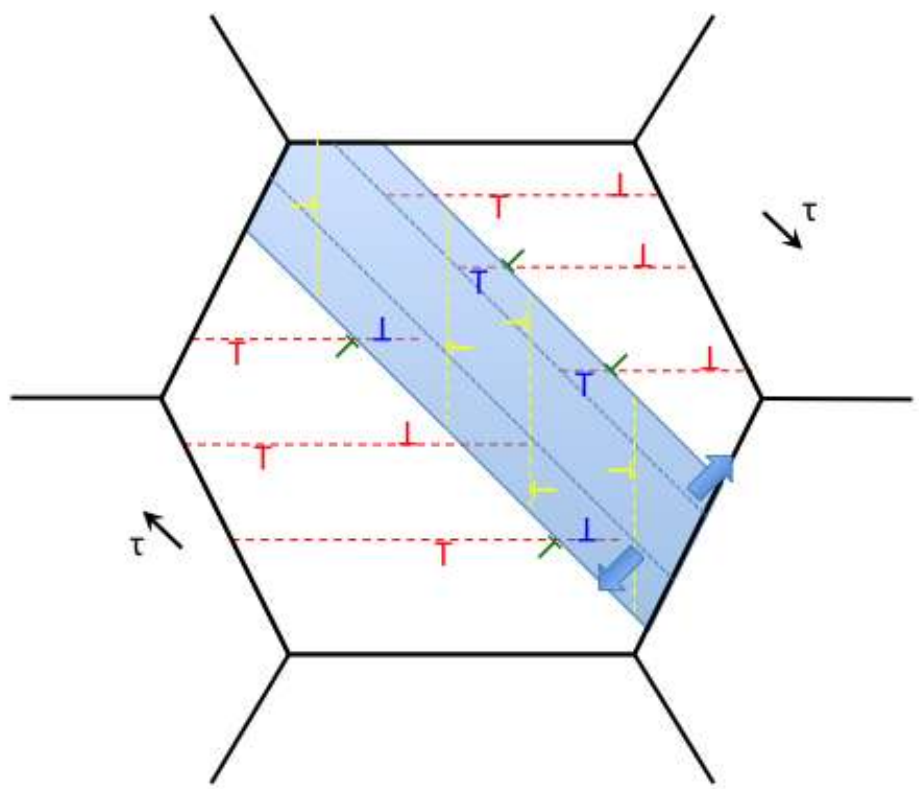

Figure 1. Schematic illustration of matrix dislocations (red) interacting with a twin boundary. Blue block arrows indicate direction of motion of the twin boundary, which has overtaken some matrix dislocations. The possible byproducts include transmuted dislocations within the twin (blue) as well as residual interfacial defects (green). Of course there are analogous interactions that can occur with dislocations within the twins (yellow) that may seek to penetrate into the matrix. Dashed lines indicate the slip planes of dislocations of the corresponding color.

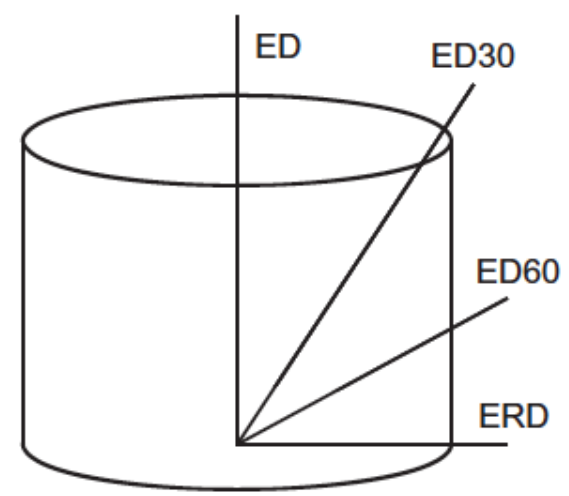

Figure 2. Schematic illustration of the four sample orientations excised from the extruded bar and tested in compression (after Oppedal et al. [1]). 

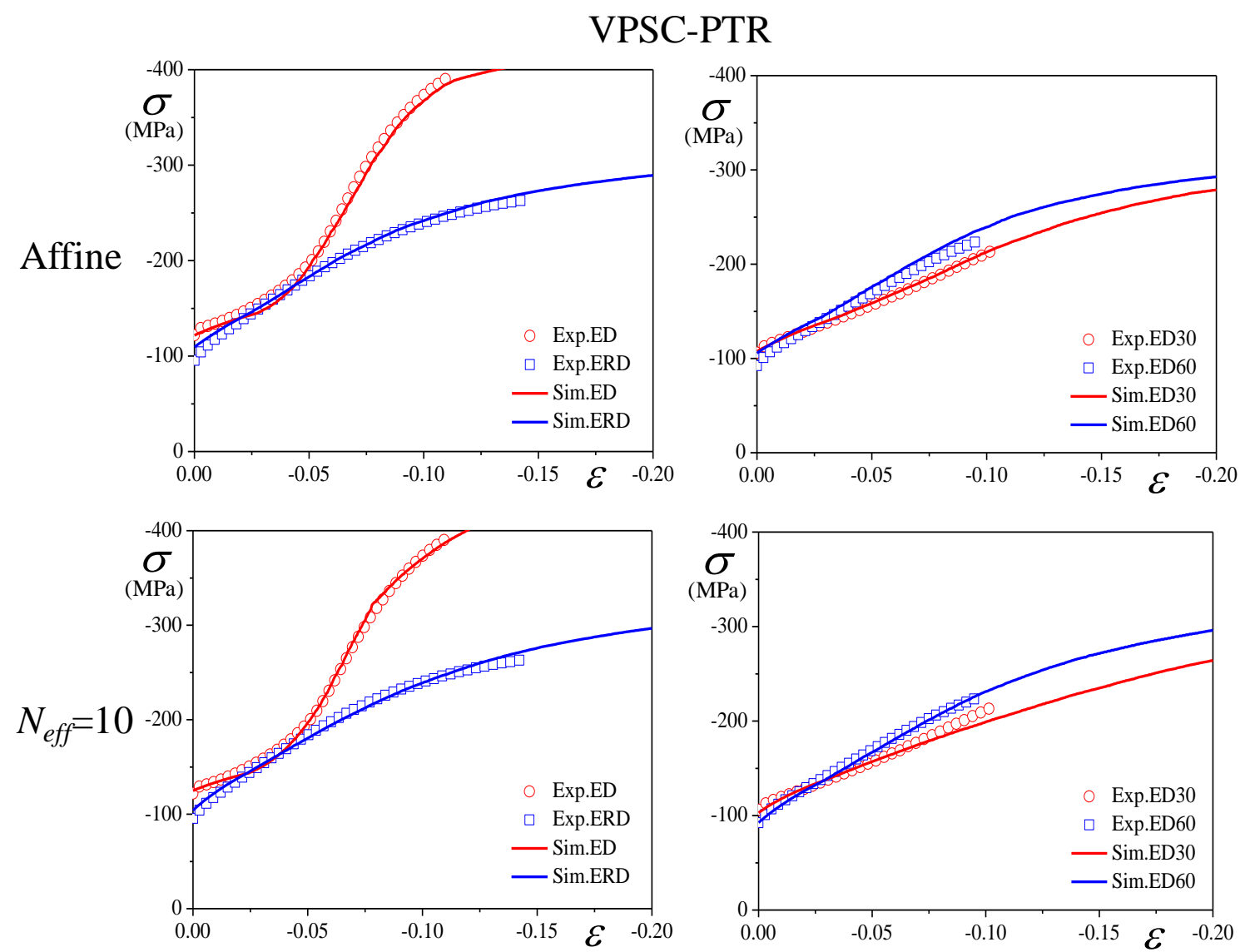

Figure 3. Experimental (Oppedal et al. [1]) and VPSC-PTR simulated compression curves for tests performed parallel to the extrusion (ED) and radial (ERD) directions (left) and two oblique directions oriented $30^{\circ}$ and $60^{\circ}$ away from the extrusion direction (see Fig. 2). 


\section{VPSC-PTR}
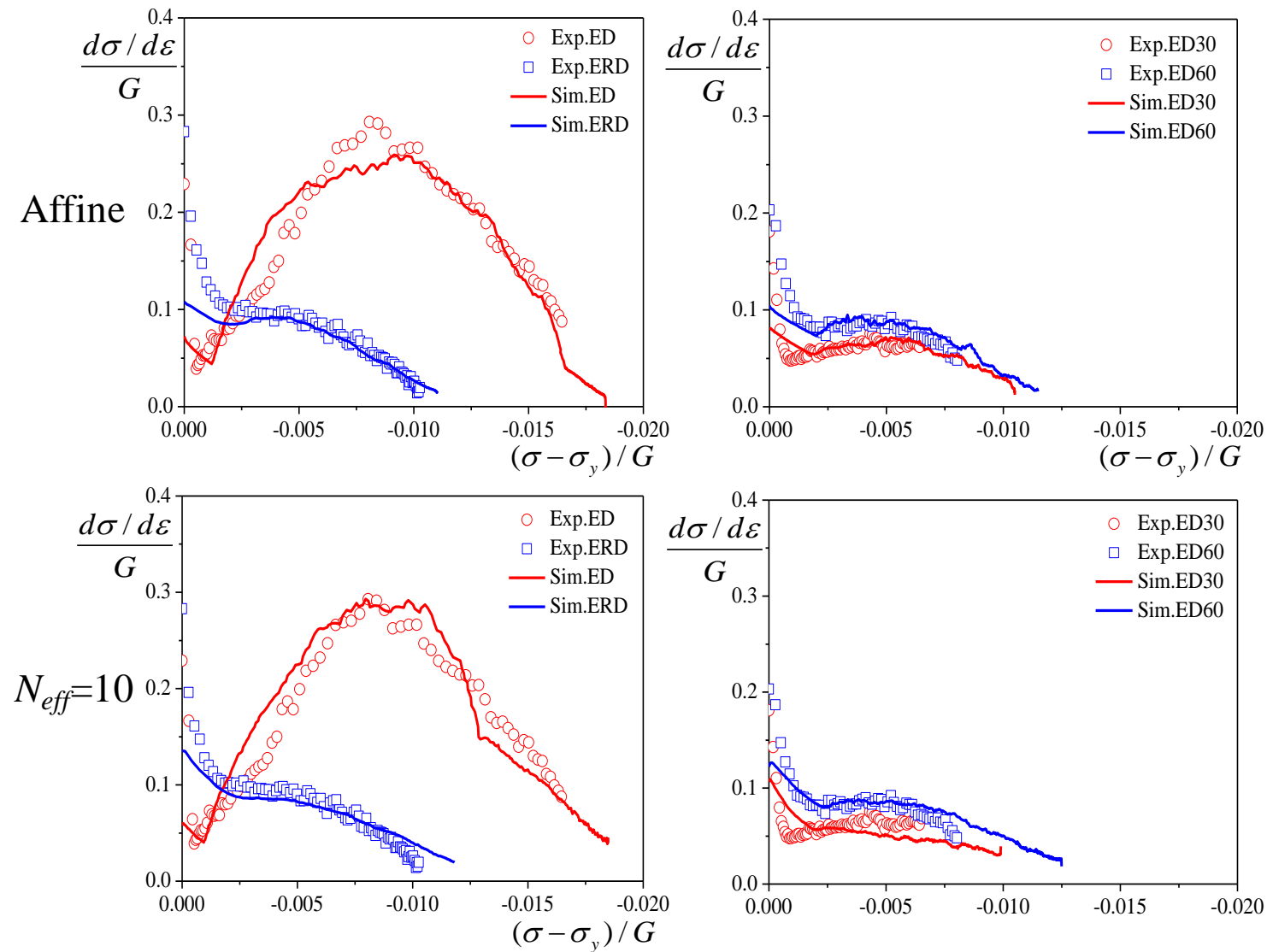

Figure 4. Experimental (Oppedal et al. [1]) and VPSC-PTR simulated normalized strain hardening rates as a function of the applied strain parallel to the extrusion (ED) and radial (ERD) directions (left) and two oblique directions oriented $30^{\circ}$ and $60^{\circ}$ away from the extrusion direction (see Fig. 2). 

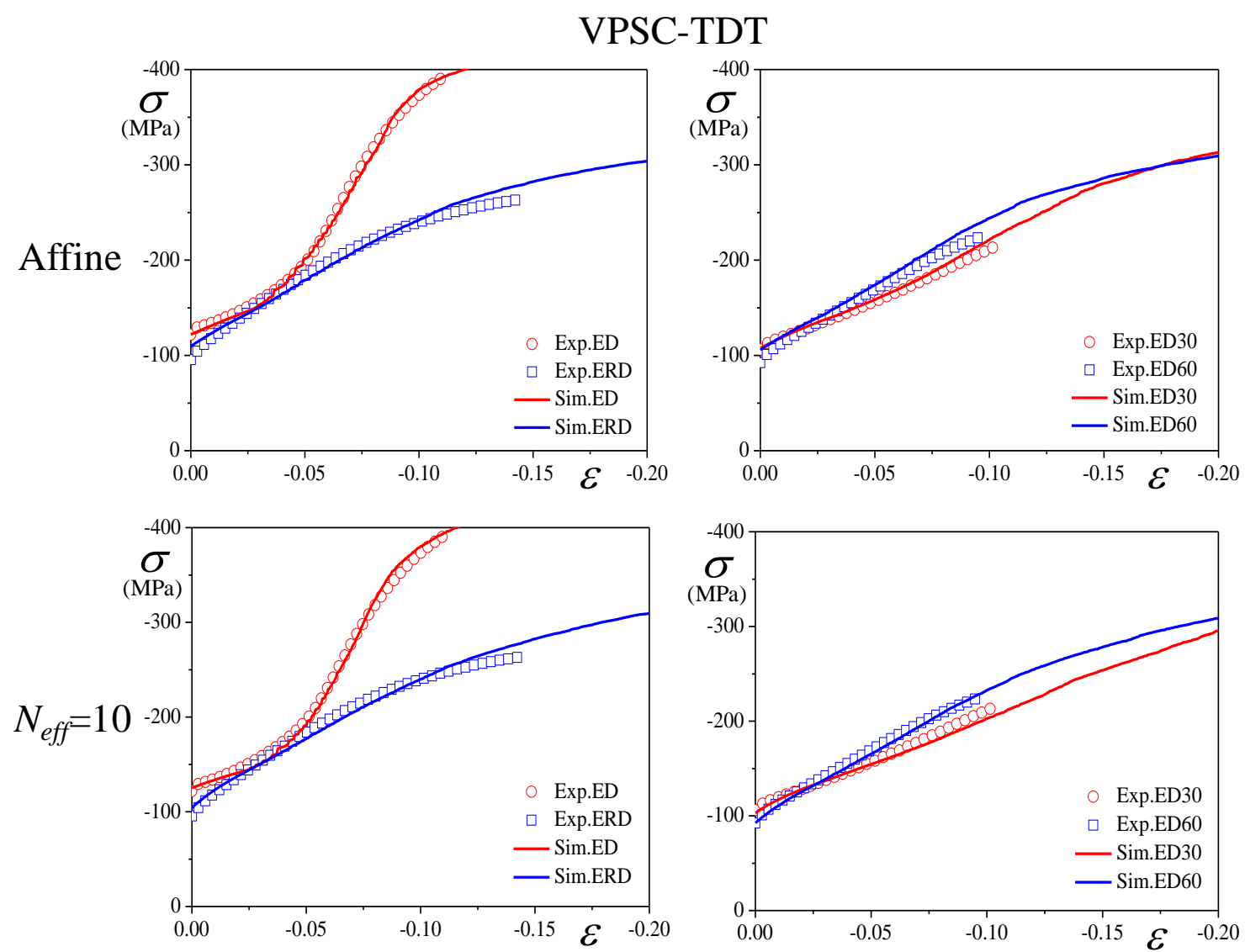

Figure 5. Experimental (Oppedal et al. [1]) and VPSC-TDT simulated compression curves, for tests performed along the 4 directions indicated in Fig. 2. 
VPSC-TDT
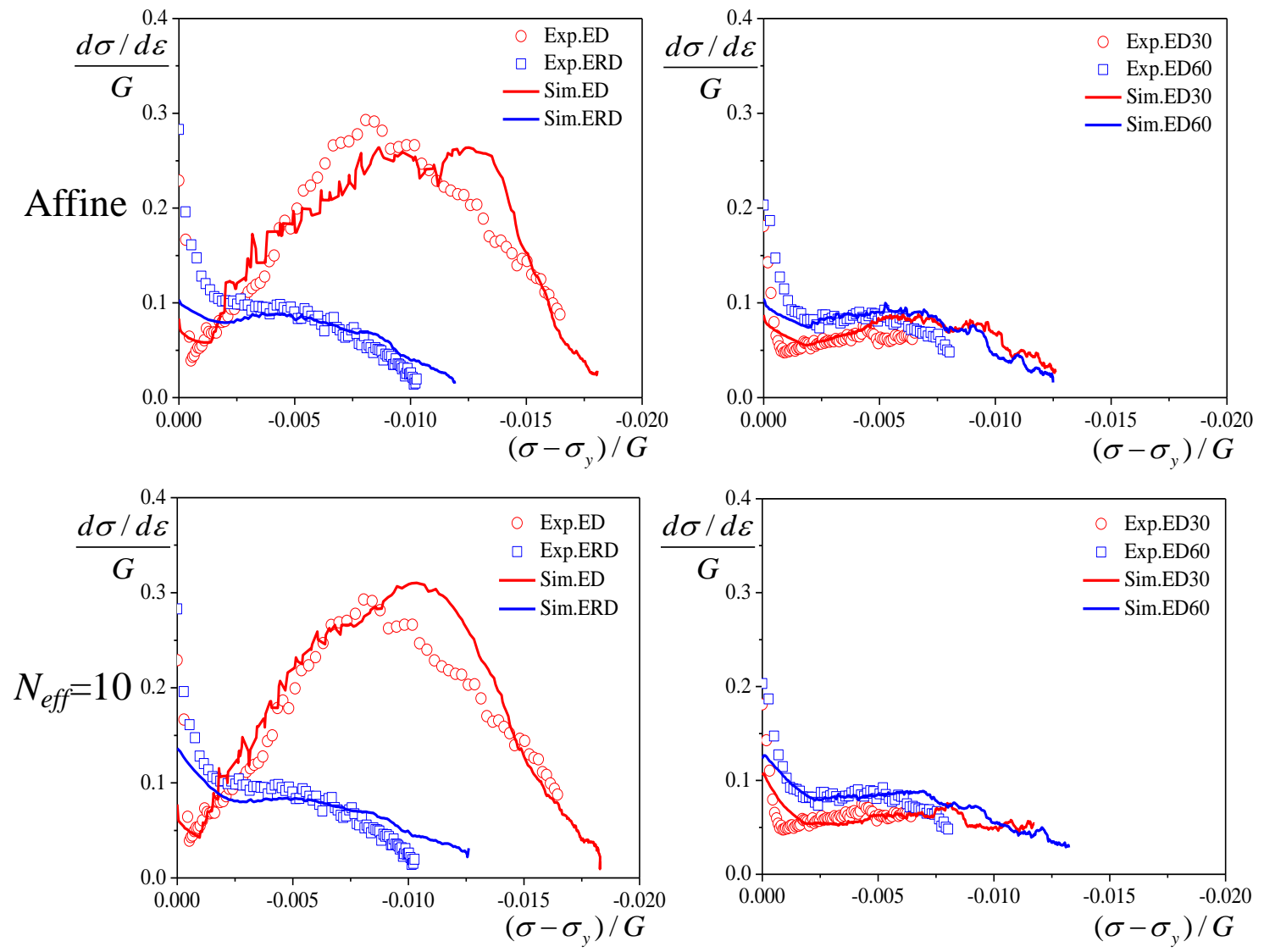

Figure 6. Experimental (Oppedal et al. [1]) and VPSC-TDT simulated normalized strain hardening rates as a function of the applied strain, for tests performed along the 4 directions indicated in Fig. 2. 


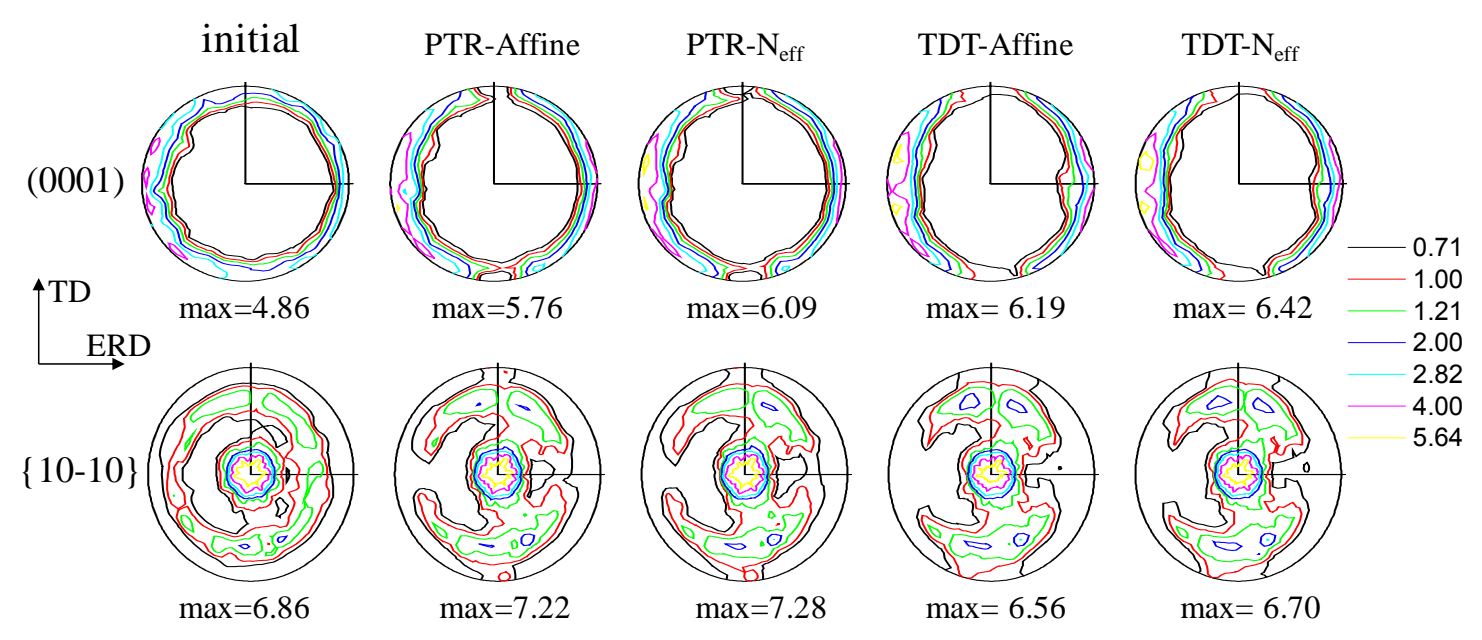

Figure 7. Crystallographic texture prior to deformation and after a compressive strain of 0.05 parallel to the radial (ERD) direction.

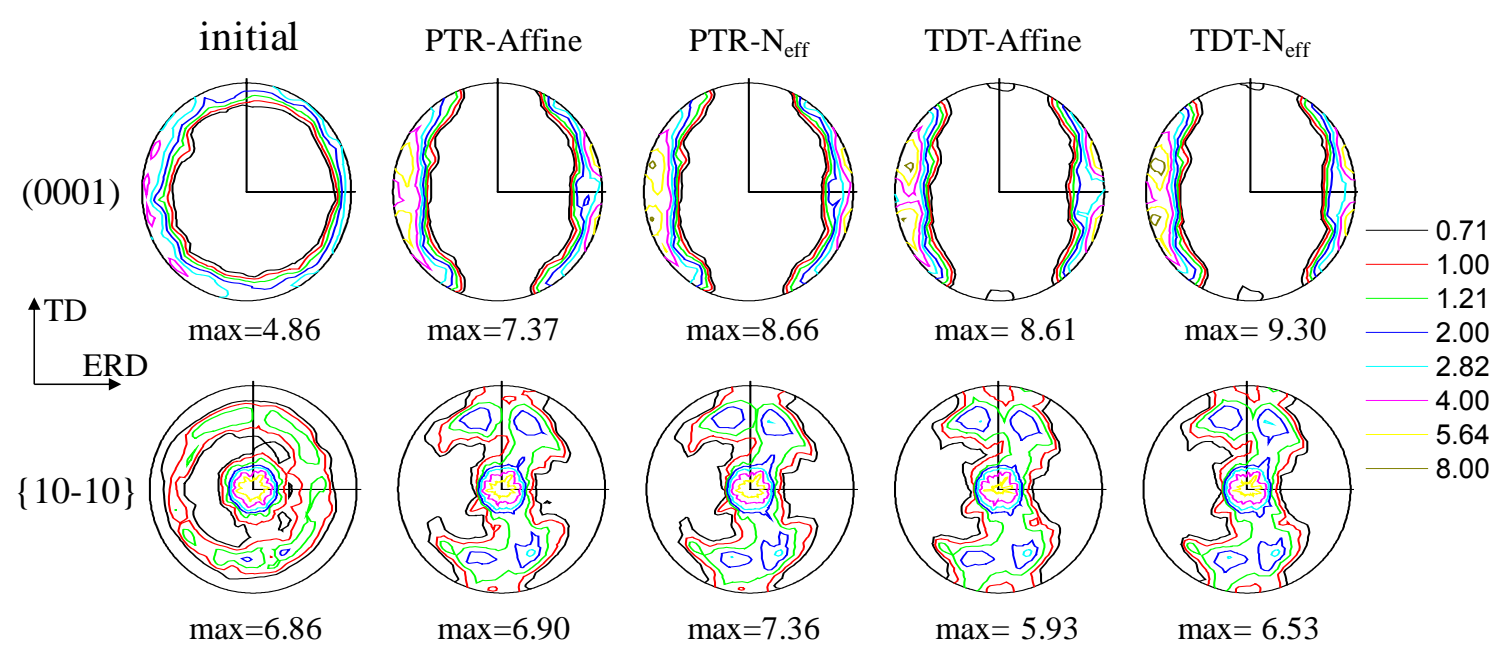

Figure 8. Crystallographic texture prior to deformation and after a compressive strain of 0.11 parallel to the radial (ERD) direction. 

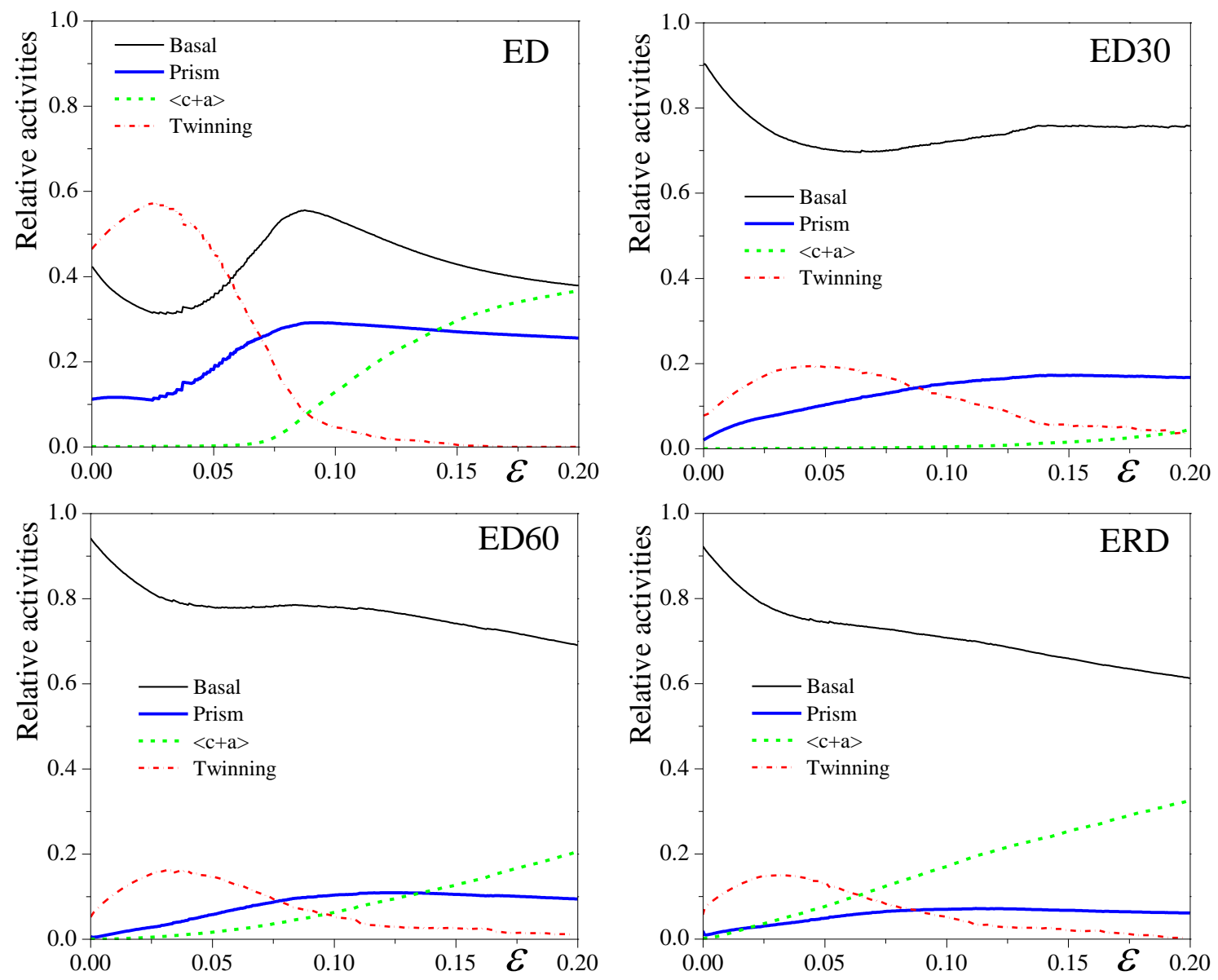

Figure 9. Deformation mechanism activity plots show how the sample orientation influences the activity of a) basal, b) prismatic, c) $\langle c+a>$ slip, and d) extension twinning during compression testing along the ED, ED30, ED60, and ERD, respectively. (The results of the VPSC-TDT, $N_{\text {eff }}=10$ calculation are presented as representative.) 

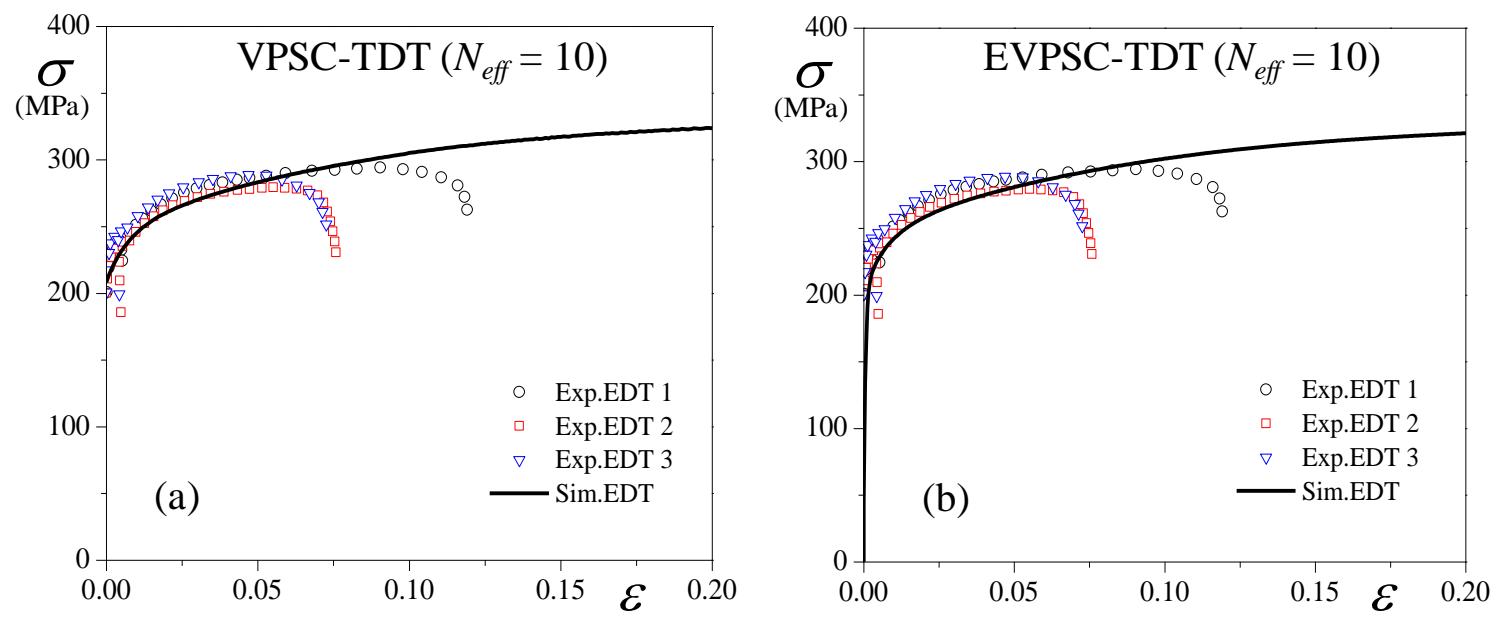

Figure 10. Experimental as well as VPSC-TDT (a) and EVPSC-TDT (b) simulated tensile curves for tests performed parallel to the extrusion (ED).
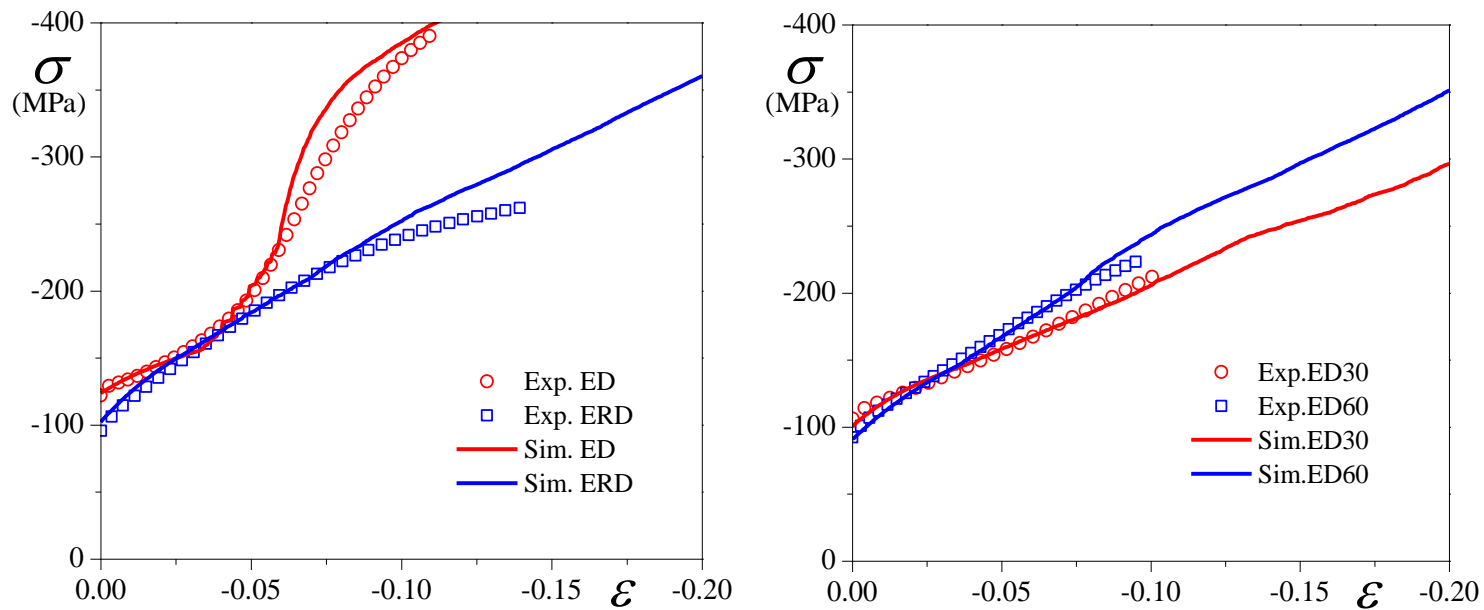

Figure 11. Experimental (Oppedal et al. [1]) and VPSC-TDT simulated compression curves, for tests performed along the 4 directions indicated in Fig. 2. 

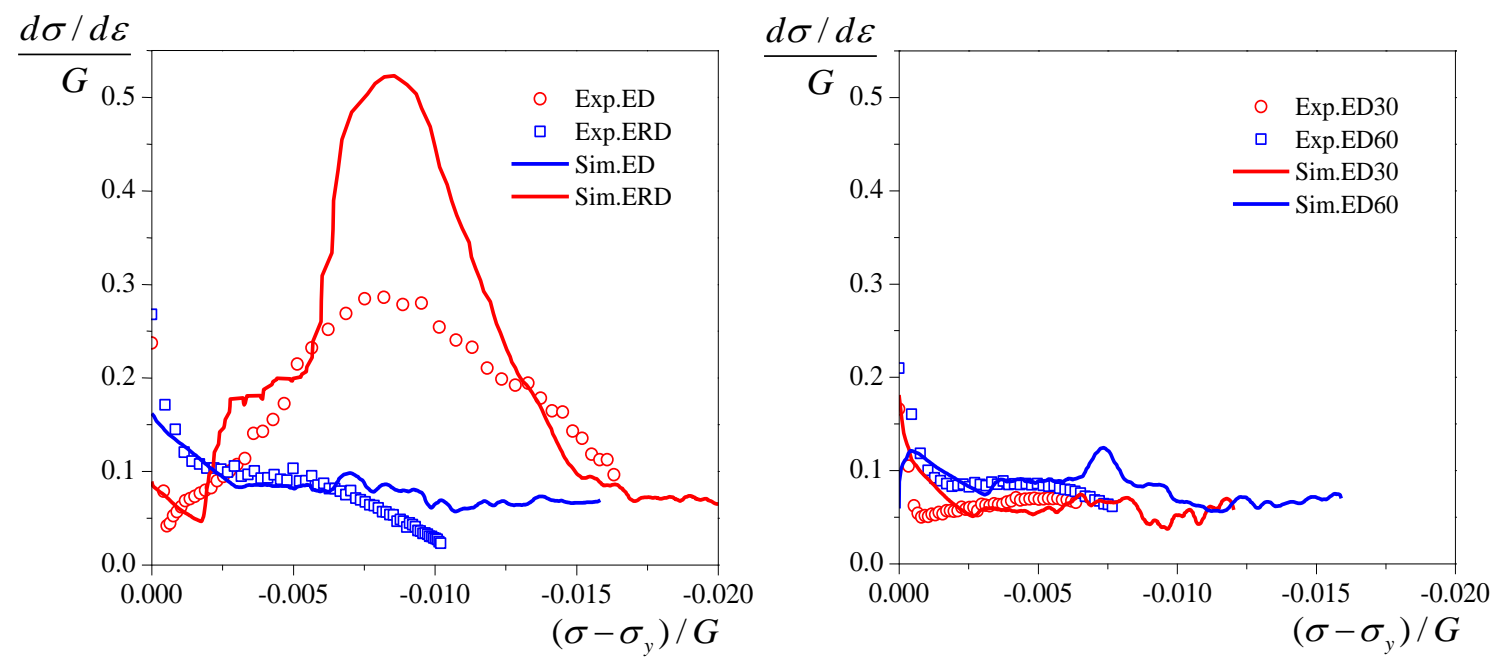

Figure 12. Experimental (Oppedal et al. [1]) and VPSC-TDT simulated strain hardening rates as a function of the normalized effective stress, for tests performed along the 4 directions indicated in Fig. 2.
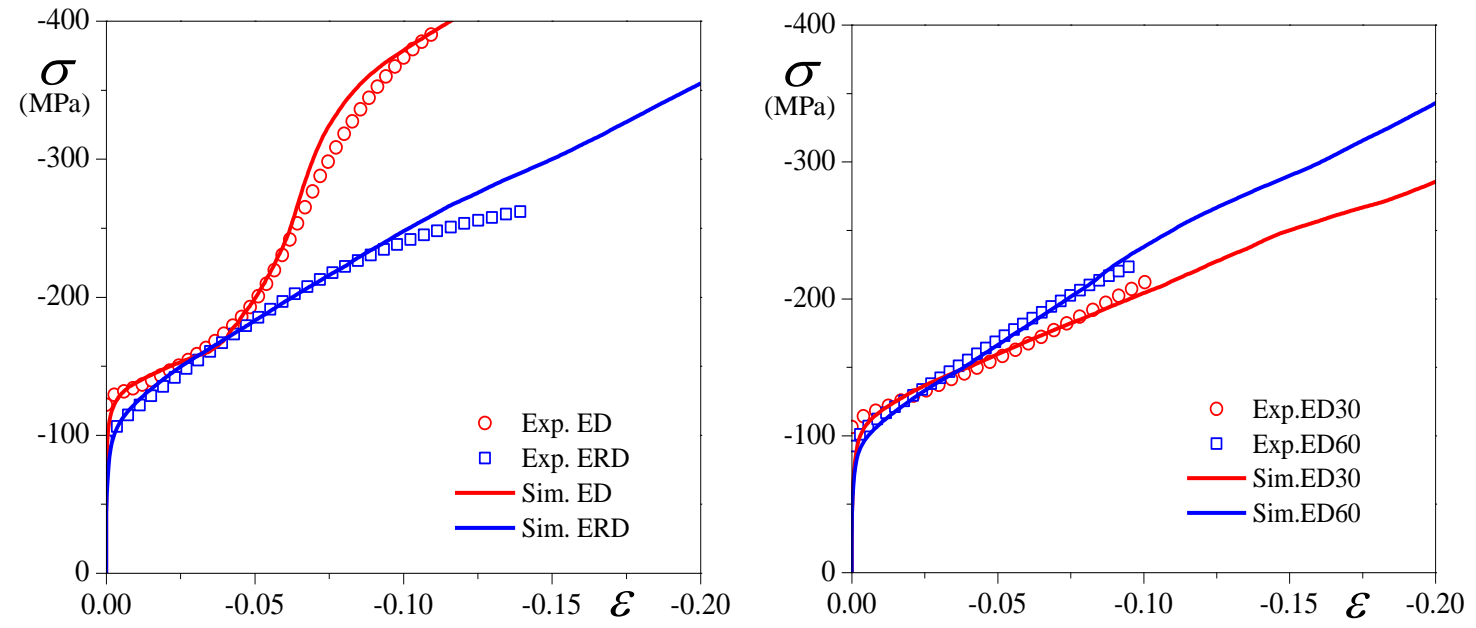

Figure 13. Experimental (Oppedal et al. [1]) and EVPSC-TDT simulated compression curves, for tests performed along the 4 directions indicated in Fig. 2. 

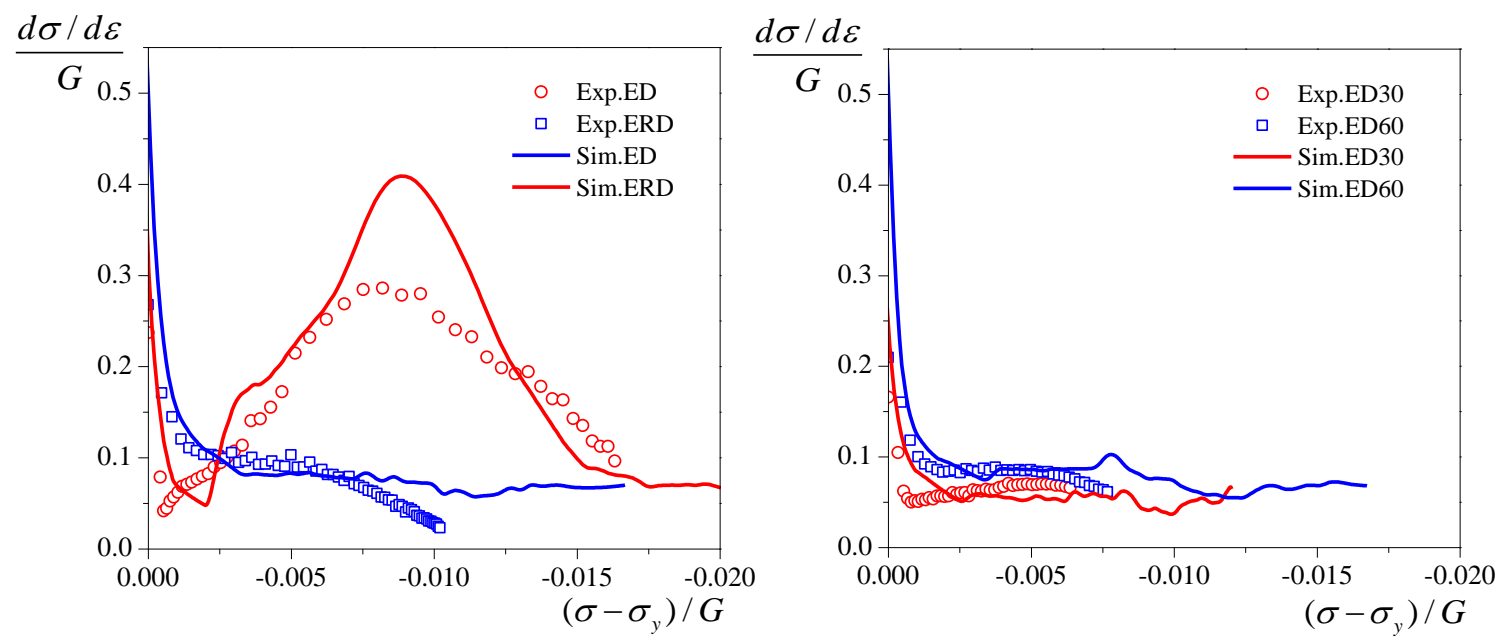

Figure 14. Experimental (Oppedal et al. [1]) and EVPSC-TDT simulated strain hardening rates as a function of the normalized effective stress, for tests performed along the 4 directions indicated in Fig. 2. 

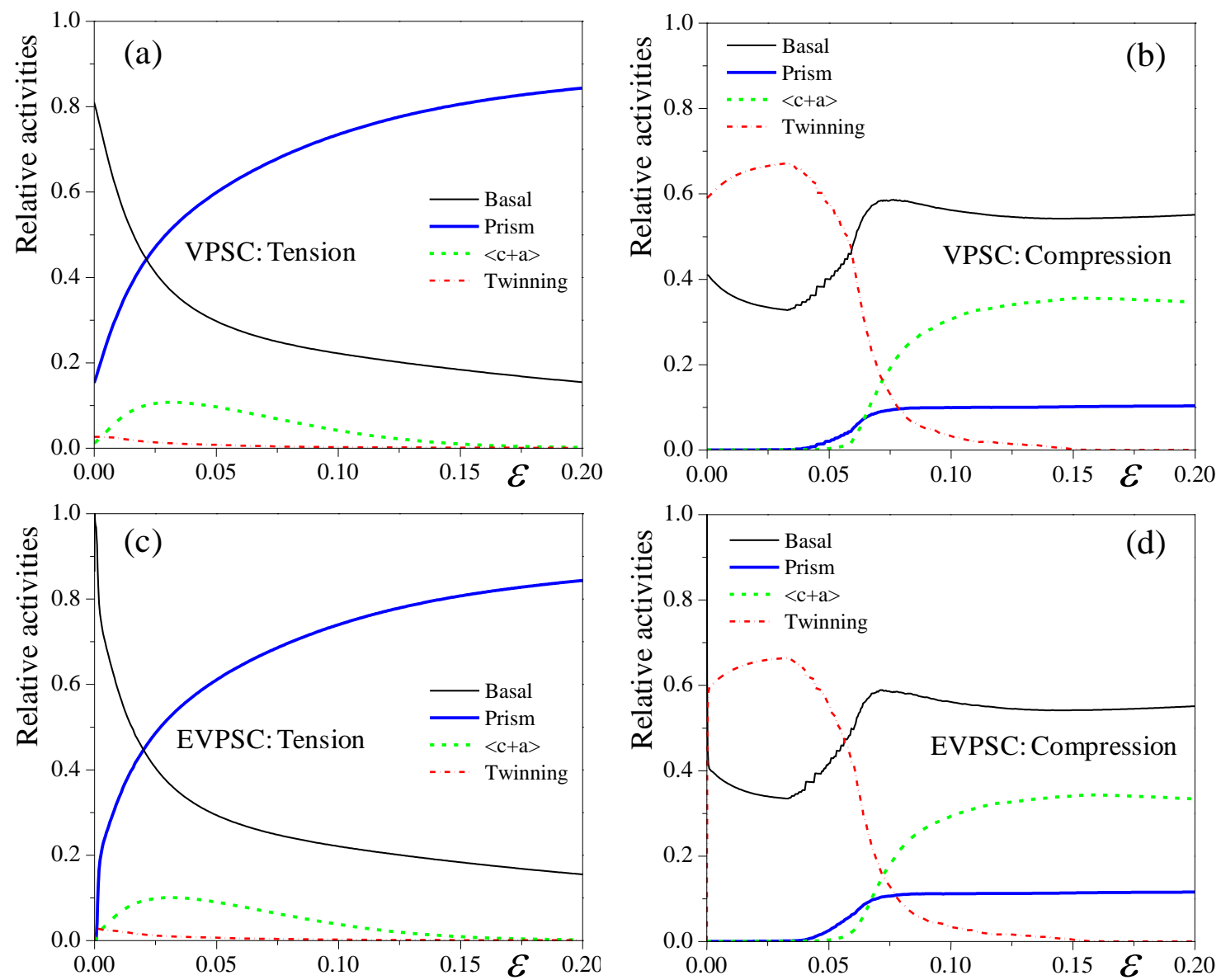

Figure 15. Deformation mechanism activity plots for tension (a \& c) and compression (b $\&$ d) strained along ED for the VPSC and EVPSC calculations, respectively. Each selfconsistent scheme employs the TDT twinning model and the $N_{\text {eff }}=10$ linearization scheme. 


\section{Tables:}

Table 1. List of values of the material parameters in the VPSC models (PTR and TDT) with the Affine and $N_{\text {eff }}=10$ self-consistent schemes. The values in bracket refer to those for the VPSC-TDT model.

\begin{tabular}{ccccccccc}
\hline Scheme & Mode & $\tau_{0}(\mathrm{MPa})$ & $\tau_{1}(\mathrm{MPa})$ & $h_{0}(\mathrm{MPa})$ & $h_{1}(\mathrm{MPa})$ & $H^{\beta \alpha}$ & $A_{1}$ & $A_{2}$ \\
\hline \multirow{2}{*}{ Affine } & Basal & 10 & 75 & 250 & 0 & 1 & & \\
PTR & Prismatic & 70 & 12 & 100 & 0 & 3 & & \\
$(T D T)$ & Pyramidal & 80 & 60 & $500(400)$ & 0 & 5 & & \\
& twinning & 55 & 0 & 0 & 0 & 1 & 0.4 & 0.7 \\
\hline \multirow{2}{*}{$N_{\text {eff }}=10$} & Basal & 18 & 70 & 220 & 0 & 1 & & \\
PTR & Prismatic & 70 & 10 & 100 & 0 & 3 & & \\
$(T D T)$ & Pyramidal & 90 & 60 & $500(400)$ & 0 & 5 & & \\
& twinning & 55 & 0 & 0 & 0 & 1 & 0.4 & 0.6 \\
\hline
\end{tabular}

Table 2. List of values of the material parameters in the VPSC-TDT model with the $N_{\text {eff }}=10$ self-consistent scheme, including ED tension.

\begin{tabular}{cccccccc}
\hline Mode & $\tau_{0}(\mathrm{MPa})$ & $\tau_{1}(\mathrm{MPa})$ & $h_{0}(\mathrm{MPa})$ & $h_{1}(\mathrm{MPa})$ & $H^{\beta \alpha}$ & $A_{1}$ & $A_{2}$ \\
\hline Basal & 18 & 40 & 240 & 0 & 1 & & \\
Prismatic & 110 & 10 & 800 & 0 & 1 & & \\
Pyramidal & 115 & 0 & 205 & 205 & 3 & & \\
twinning & 50 & 0 & 45 & 45 & 1 & 0.6 & 0.75 \\
\hline
\end{tabular}

Table 3. List of values of the material parameters in the EVPSC-TDT model with the $N_{\text {eff }}=10$ self-consistent scheme, including ED tension.

\begin{tabular}{cccccccc}
\hline Mode & $\tau_{0}(\mathrm{MPa})$ & $\tau_{1}(\mathrm{MPa})$ & $h_{0}(\mathrm{MPa})$ & $h_{1}(\mathrm{MPa})$ & $H^{\beta \alpha}$ & $A_{1}$ & $A_{2}$ \\
\hline Basal & 18 & 40 & 240 & 0 & 1 & & \\
Prismatic & 110 & 10 & 800 & 0 & 1 & & \\
Pyramidal & 115 & 0 & 205 & 205 & 3 & & \\
twinning & 52 & 0 & 55 & 55 & 1 & 0.6 & 0.65 \\
\hline
\end{tabular}

